International Journal of Wireless \& Mobile Networks (IJWMN) Vol. 3, No. 4, August 2011

\title{
Performance Analysis of ON-Demand Routing Protocols FOR VEHICULAR AD-HOC NETWORKS
}

\author{
A. Shastri ${ }^{1}$, R. Dadhich ${ }^{2}$, Ramesh C. Poonia ${ }^{3}$ \\ ${ }^{1}$ Prof. \& Vice-Chancellor, Banasthali University, Banasthali, India. \\ adityashastrieyahoo.com \\ ${ }^{2}$ Associate Prof. \& Head Department of MCA, Govt. College of Engineering, Ajmer, \\ India. \\ reena.dadhich@gmail.com \\ ${ }^{3}$ Research Scholar, Apaji Institute of Mathematics \& Applied Computer Technology, \\ Banasthali University, Banasthali, India. \\ rcpooniadyahoo.com
}

\begin{abstract}
Vehicular Ad Hoc Networks (VANETs) are a peculiar subclass of mobile ad hoc networks that raise a number of technical challenges, especially from the point of view of their mobility models. Currently, the field of VANETs has gained an important part of the interest of researchers and become very popular. More specifically, VANETs can operate without fixed infrastructure and can survive rapid changes in the network topology. The main method for evaluating the performance of routing protocols for VANETs by Network Simulator-2.34. This paper is subjected to the on-demand routing protocols with identical loads and evaluates their relative performance with respect to the two performance context: average End-toEnd delay and packet delivery ratio. We investigated various simulation scenarios with varying pause times, connections and no. of nodes particularly for AODV and DSR. We will also discuss briefly about the feasibility of VANETs in respect of Indian automotive networks.
\end{abstract}

\section{KEYWORDS}

VANETs, NS-2.34, V2V, V2R, AODV, DSR

\section{INTRODUCTION}

Ad-hoc network is an infrastructureless network with no fixed routers, hosts or wireless base stations. Vehicular Ad-hoc Networks (VANETs) represent a rapidly emerging, particularly challenging class of Mobile Ad Hoc Networks (MANETs) [1]. VANETs are distributed, self organizing communication networks built up by moving cars as nodes in a network to create a very high mobility of nodes and limited degrees of freedom in nodes movement patterns. It turns every participating car into a wireless router or node, allowing cars approximately 100 to 300 meters of each other to connect and in turn, create a network with a wide range. VANET integrates on multiple ad-hoc networking technologies such as Wi-Fi IEEE $802.11 \mathrm{~b} / \mathrm{g}$, WiMAX IEEE 802.16, Bluetooth, IRA, ZigBee for easy, accurate, effective and simple communication between vehicles on dynamic mobility [2]. Some Important features of VANETs are: Vehicles move in more predictable fashion, portability issues are not a problem (computational power, energy, antennas, etc.), location information can be assumed, the nodes in a VANET are vehicles and road side units, the movement of these nodes is very fast, the motion patterns are restricted by road topology, vehicle acts as transceiver i.e. sending and receiving at the same time while creating a highly dynamic network, Which is used as part of automotive electronics to identify an optimally minimal path for navigation with minimal traffic intensity. The system

DOI : 10.5121/ijwmn.2011.3407 
can also be used as a city guide to locate and identify landmarks in a new city. Vehicles are enabled to communicate among themselves (vehicle-to-vehicle, $\mathrm{V} 2 \mathrm{~V}$ ) and via roadside access points (vehicle-to-roadside, V2R) [3]. Vehicular communication is expected to contribute to safer and more efficient traffic by providing timely information to drivers, and also to make travel more convenient, figure 1.

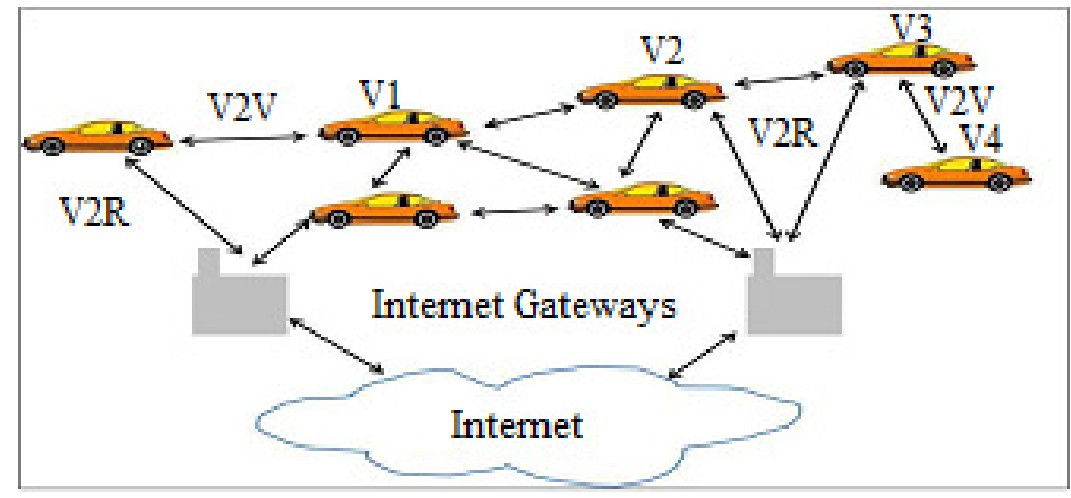

Figure 1. The scenario of VANETs

For communication in vehicular ad hoc networks, On Demand Routing Protocols is required. Currently there is lot of research work in the field of VANETs for several scenarios [4]. Recent research [5],[6] has focused on topology related problems such as range optimization, routing mechanisms, or address systems, as well as security issues like traceability or encryption. Concerning to these facts there is an urgent need of protocols and technologies to develop VANETs for the safety of human beings. The main goal of this paper is to focus on analyzing the performance of routing protocols in VANETs so that it will help to researchers to adopt the application for their contribution in VANETs.

The complete paper is organized as follows: In Section 2, we shortly provide some related work, while in Section 3, we will discuss in brief about the feasibility of VANTEs in Indian Automotive Networks, Section 4 presents the routing protocols in VANTEs. Evaluation parameters for routing protocols are discussed in Section 5. The simulation results and performance analysis is done in section 6and the paper concludes in Section 7.

\section{RELATED WORK}

In this section we will discuss in brief the initial development that were carried in creating a simulation that was aimed at testing VANETs, e.g. CanuMobiSim, NS-2 and NS-3, GlomoSim, QualNet, MOVE, TraNs, VanetMobiSim, NCTUns [7]-[9] and many more. The very first work related to the title of the paper was proposed by Ott and Kutscher's seminal in [10]. They discussed about the drive-thru Internet problem and performed a detailed experimental analysis of opportunistic Internet access in vehicles. Jaap et al. [11] evaluated AODV, DSR, FSR, and TORA in city traffic scenarios. The simulation shows that AODV has the best performance and lowest control overhead. It is followed by FSR, DSR, and then TORA. DSR suffers from a very high delay because source routes change continuously due to high mobility. Its route overhead is comparable to FSR yet higher than AODV since DSR keeps route information with in the packet header. The common characteristic among all four routing protocols is that performance degrades as network densities increase, indicating their scalability problem.

There are many papers [12],[13] focusing on new routing protocols to VANETs, in order to achieve increased communication reliability in some mobility scenarios (highway and Urban area) or particular application. 
International Journal of Wireless \& Mobile Networks (IJWMN) Vol. 3, No. 4, August 2011

\section{THE FEASIBILITY OF VANETS}

The Automotive in India is one of the largest fields in the world and one of the fastest growing globally. India manufactures over 17.5 million vehicles (including 2 wheeled and 4 wheeled) and exports about 2.33 million every year. India's passenger car and commercial vehicle manufacturing industry is the seventh largest in the world, with an annual production of more than 3.7 million units in 2010. According to recent reports, India is set to overtake Brazil to become the sixth largest passenger vehicle producer in the world [14]. The feasibility of VANET in a certain city as well as on highways has to be justified. First, there needs to be an adequate number of vehicles in the city road and highway at any given time in order for ad hoc networking among vehicles to be possible. The density of vehicles is $\lambda$ vehicles $/ \mathrm{km}^{2}$. Furthermore, any vehicle should be within range of at least one other vehicle in order for a link to be established and multihop routing to prove practical [15]. According to the practical situation, we make the line-of-sight (LOS) distance $r$ and the geometrical area of the communication zone $\mathrm{S}$ is calculated using

$$
\mathrm{S}=\pi \mathrm{r}^{2}
$$

Using the Possion distribution, the probability of the number of vehicle $\mathrm{n}$ in the region $\mathrm{S}$ can be worked out using

$$
p(n)=\frac{(\lambda S)^{n}}{n !} e^{-\lambda S}
$$

Thus, the probability of finding at least $\mathrm{k}$ vehicles in area $\mathrm{S}$ is

$$
q(k)=1-\sum_{n=0}^{k-1} \frac{(\lambda S)^{n}}{n !} e^{-\lambda S}
$$

\section{ROUTING PROTOCOLS IN VANETS}

Standard As a special type of network, Vehicular Ad hoc Networks (VANETs) have received increasing research attention in recent years. There are many active research projects [19] concerned with VANETs. Vehicular ad hoc networks are wireless networks that use multi-hop routing instead of static networks infrastructure to provide network connectivity. VANETs have applications in rapidly deployed and dynamic military and civilian systems. The network topology in VANETs usually changes with time. Therefore, there are new challenges for routing protocols in VANETs since traditional routing protocols may not be suitable for VANETs [16],[17]. Researchers are designing new VANETs routing protocols, comparing and improving existing ones by using simulations. This work is an attempt towards a comprehensive performance evaluation of two commonly used mobile ad hoc routing protocols (DSR and AODV).

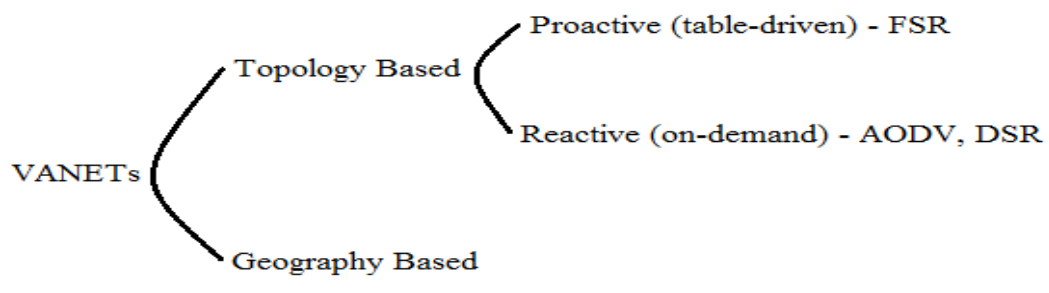

Figure 2. Taxonomy of Routing Protocols in VANET 
International Journal of Wireless \& Mobile Networks (IJWMN) Vol. 3, No. 4, August 2011

Figure 2 illustrates the taxonomy of these VANET routing protocols [18],[19] which can be classified as topology-based and geographic (position-based) in VANET. Topology-based routing uses the information about links that exist in the network to perform packet forwarding. Geographic routing uses neighbouring location information to perform packet forwarding [20]. Since link information changes in a regular basis, topology-based routing suffers from routing route breaks.

\subsection{Topology-based Routing Protocols}

These routing protocols use link information that exists in the network to perform packet forwarding. They can further be divided into proactive (table-driven) and reactive (on-demand) routing [21].

\subsubsection{Proactive (table-driven) Protocols}

Table-driven routing protocols [22] try to maintain consistent, up-to-date routing information from each node to every other node. Network nodes maintain one or many tables for routing information. Nodes respond to network topology changes by propagating route updates throughout the network to maintain a consistent network view. The advantage of the proactive routing protocols is that there is no route discovery since route to the destination is maintained in the background and is always available upon lookup. Despite its good property of providing low latency for real-time applications, the maintenance of unused paths occupies a significant part of the available bandwidth, especially in highly mobile VANETs [23],[24].

\subsubsection{Reactive (On-Demand) Protocols}

Reactive routing opens [25] a route only when it is necessary for a node to communicate with another node. It maintains only the routes that are currently in use, thereby reducing the burden on the network. Reactive routings typically have a route discovery phase where query packets are flooded into the network in search of a path. The phase completes when a route is found.

\subsection{Geographic Based}

Geography based routing is basically a location based multicast routing. Its objective is to deliver the packet from source node to all other nodes within a specified geographical region (Zone of Relevance ZOR) [20]. In Geography based routing vehicles outside the ZOR are not alerted to avoid unnecessary hasty reaction. It is considered as a multicast service within a specific geographic region.

\section{EVALUATION PARAMETERS FOR ROUTING PROTOCOLS}

In this paper, we evaluated the performance of two widely used ad hoc network routing protocols using packet-level simulation using the latest simulation environment NS-2.34 [26],[27] under working Windows 7. The simulation characteristics used in our research, include parameters described as follows for detailed performance evaluation of any networking protocol and the simulation parameters are summarized in Table 1.

- Packet Delivery Fraction (PDF): It represents the ratio of the data packets received at destination and the delivered packets.

- End-to-end Average Delay: It indicates the propagation and transfer delay.

- Received packets: It measures the numbers of received packets.

- Reception time of the first packet: This parameter informs us about the time it takes to establish the connection for each of the protocols. Dropped Packets: It represents those packets which fail to reach their destination. 
International Journal of Wireless \& Mobile Networks (IJWMN) Vol. 3, No. 4, August 2011

- Number of Hops: It refers to the number of hops that the packets need to reach their destination.

Table 1. Simulation Parameters

\begin{tabular}{|l|l|}
\hline Parameters & Specifications \\
\hline Network Simulator & NS-2.34 \\
\hline Routing Protocols & AODV, DSR \\
\hline Simulation Area & $500 \mathrm{~m}$ x 500m \\
\hline Propagation Model & Two-ray ground reflection model \\
\hline Number of Vehicles & 50 \\
\hline Antenna model & Omnidirectional \\
\hline MAC Protocol & IEEE 802.11 \\
\hline Channel Type & Wireless channel \\
\hline Traffic Type & Constant Bit Rate $(\mathrm{CBR})$ \\
\hline Source/Destination & Random \\
\hline Max. Speed & $20 \mathrm{~m} / \mathrm{s}$ \\
\hline Simulation Time & $200 \mathrm{~s}$ \\
\hline Data Rate & $4 \mathrm{Mbps}$ \\
\hline Seed & 1 \\
\hline Pause Time & $50,100,150,200,250,300,350,400,500$ \\
\hline Connections & $10,20,30,40,50,60,70,80,90,100$ \\
\hline
\end{tabular}

In this table, the variables along with their appropriate values are specifying the simulation's parameters for all the density phases of movement model.

\section{SIMULATION RESULTS AND PERFORMANCE ANALYSIS}

Figure 3 shows the behaviour by throughput of AODV and DSR.

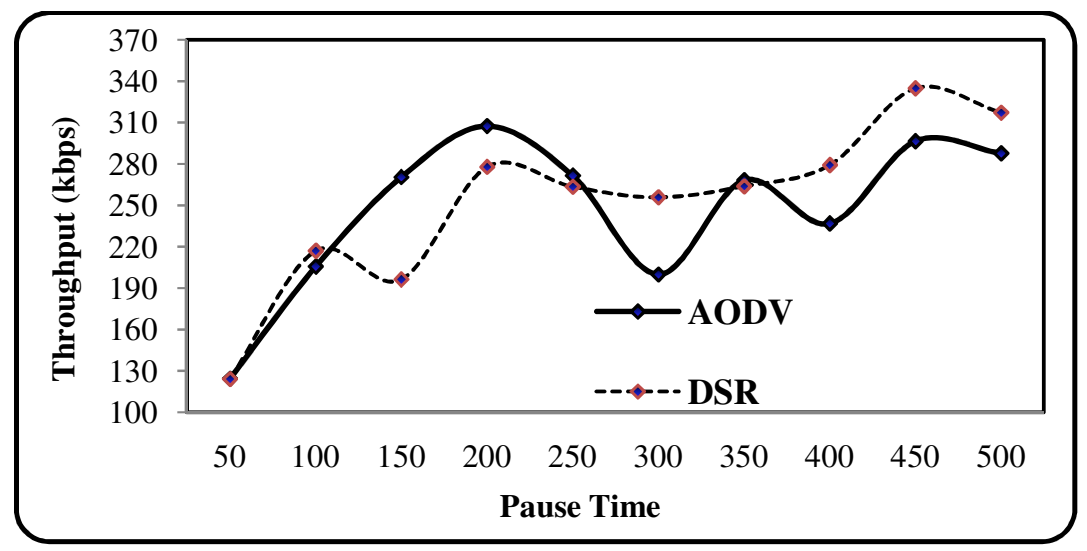

Figure 3. Throughput of AODV and DSR

First, we will compare two protocols under the above simulation environment. For the simulations, the same movement models are used, the number of traffic sources is fixed at 50 , the maximum speed of the nodes is set to $20 \mathrm{~m} / \mathrm{s}$ and the pause time is vary as $50,100,150,200$, $250,300,350,400,450$ and 500. Figure 4 highlights the relative performance of the two routing protocols. It shows that there is an increasing packets loss in AODV as compared to DSR when as the pause time increases. 


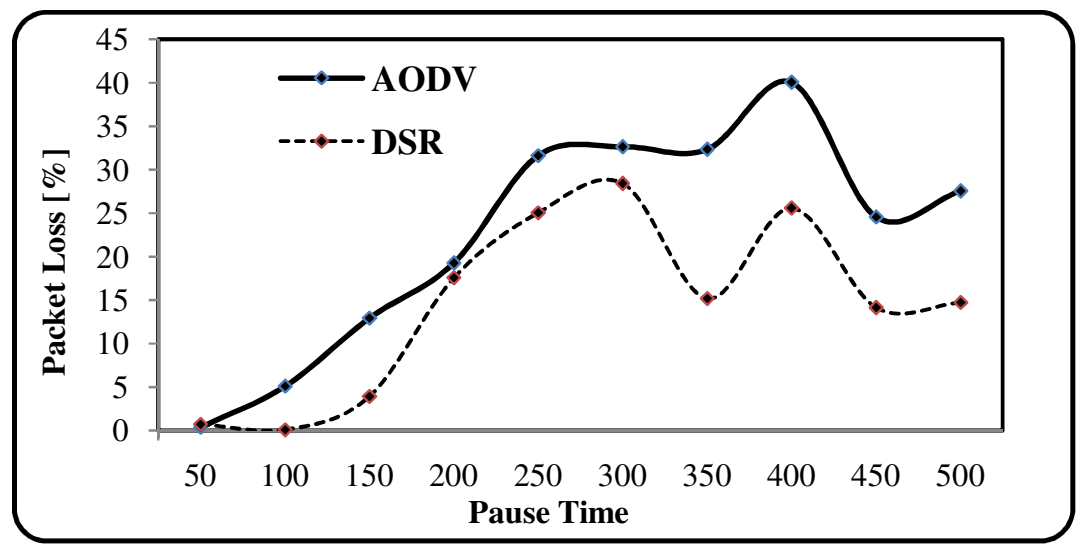

Figure 4. Packet Loss of AODV and DSR

\subsection{Packet delivery Comparison}

Figure 5 shows that Packet Delivery Fraction (PDF) for the same movement models are used, the number of traffic sources is fixed at 50, the maximum speed of the nodes is set to $20 \mathrm{~m} / \mathrm{s}$ and the pause time is vary as $50,100,150,200,250,300,350,400,450$ and 500.The packet delivery fractions for AODV and DSR are almost similar with 50, 200, 250, 300 and 350 pause times shown in figure 5. However, with 100, 150, 200, 400, 450 and 500 pause times, AODV outperforms DSR by about 10 percent.

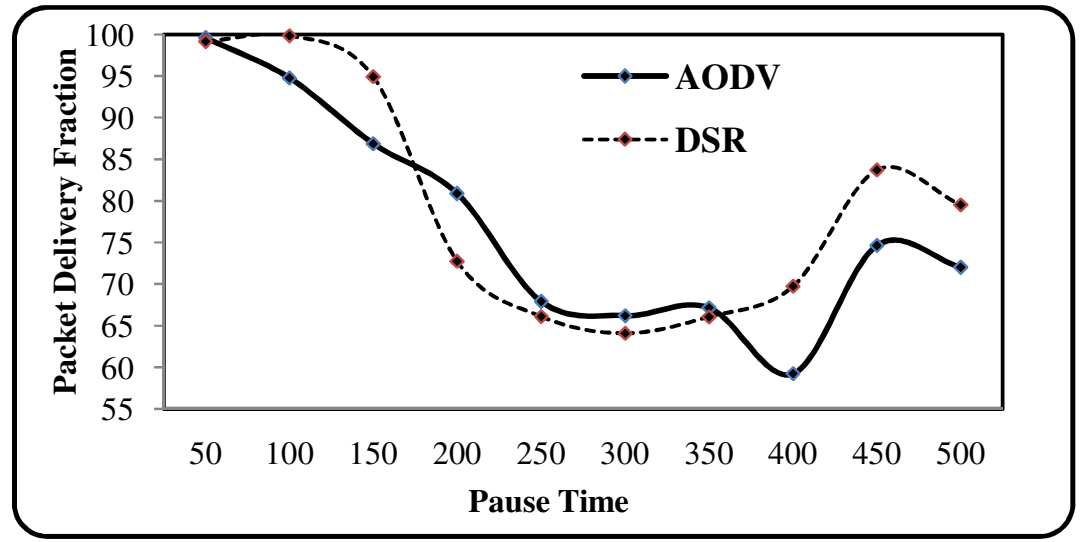

Figure 5. Packet Delivery Fraction (PDF) of AODV and DSR

\subsection{Average End-to-End delay in Packet Delivery}

The average end-to-end delay in packet delivery is higher in DSR as compared to AODV, figure 6. They are little and similar in the case of 100, 150, 400, 450 and 500 pause times. When the pause time increases to 200 , the transmission delay of data packet increases greatly and that of AODV is obviously lower than DSR. The DSR becomes better than AODV when pause time becomes longer. 


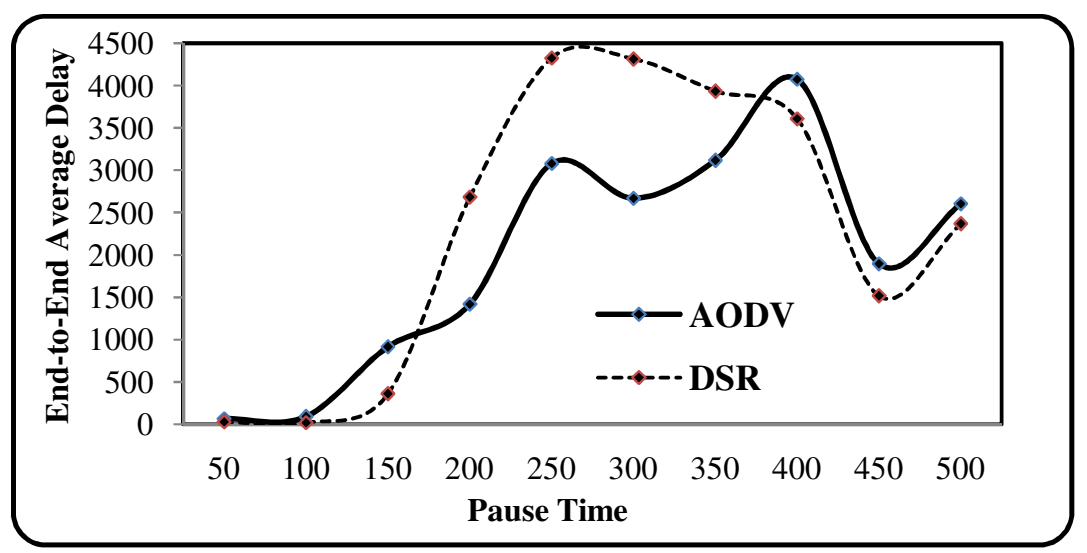

Figure 6. End-to-End average Delay of AODV and DSR

\subsection{Received Packets}

Similar to above observations for packet delivery ratio (PDR), elevate percentage means higher performance. In view of this fact, at all simulated pause times, the DSR delivers successfully a slightly greater performance of packets than the AODV so that DSR remained the highly acceptable approaches for the movement model. Figure 7 shows in all cases of received packet delivery with respect to pause times. It can be observed that the behaviour of DSR is better than AODV.

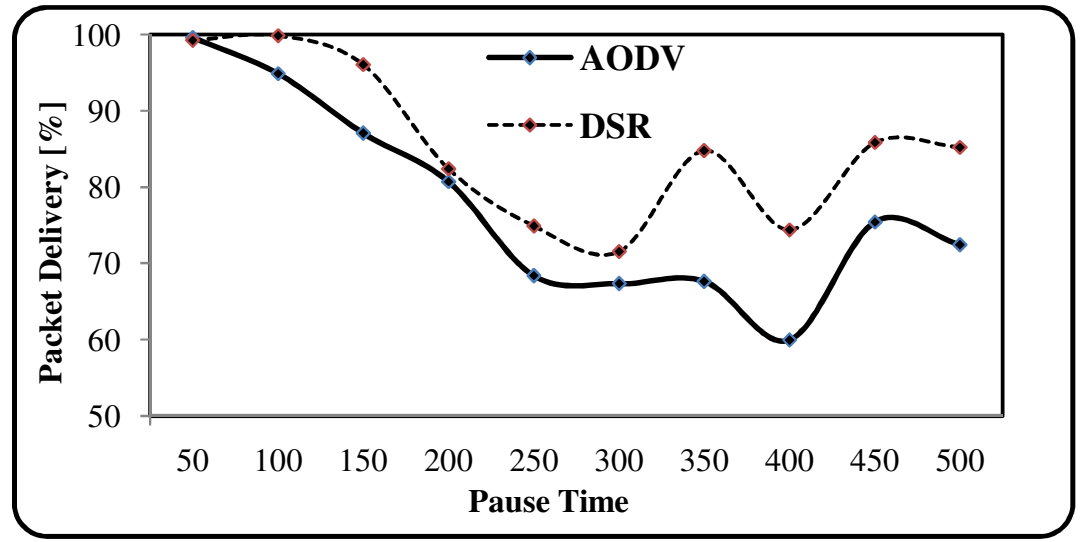

Figure 7. Received Packet Delivery of AODV and DSR

\section{CONCLUSION AND FUTURE WORK}

This paper presents a performance comparison between two reactive routing protocols for vehicular ad hoc networks: ad hoc on demand distance vector (AODV), dynamic source routing (DSR). Both protocols were simulated using the tool NS-2.34 and were compared in terms of packet loss ratio, end to end delay, throughput with 50 nodes, 20 speed and varying pause time 50 to 500 and making connections 10 to 100 . From the simulation results in section V we can conclude that increase in the pause times increase both Packet Loss and Average End-to-End Delay but decrease the Packet Delivery Fraction. In the future, extensive complex simulations could be carried out using other existing performance metrics, in order to gain a more in-depth performance analysis of the ad hoc routing protocols. Other new protocols performance could be studied too. We can also analyse and evaluate in depth of VANETs in respect of Indian automotive networks. 
International Journal of Wireless \& Mobile Networks (IJWMN) Vol. 3, No. 4, August 2011

\section{REFERENCES}

[1] Tarik Taleb, Ehssan Sakhaee, Abbas Jamalipour, etl.,( 2007) "A Stable Routing Protocol to Support ITS Services in VANET Networks", IEEE Transactions on Vehicular Technology,56(6): 33373347.

[2] Pranav Kumar Singh, Kapang Lego, Themrichon Tuithung, (March 2011) "Network Centric Approach using MOVE \&Application Centric Approach using TraNS for protocols and safety in VANET", International Journal of Research and Reviews in Computer Science (IJRRCS), Vol. 2, No. 1.

[3] C2C-CC: CAR 2 CAR Communication Consortium, http://www.car-to-car.org/ Visited in May 2011.

[4] Xin Jin Weijie Su Yan Wei, (May 2011) "A study of the VANET connectivity by percolation theory", Consumer Communications and Networking Conference (CCNC), IEEE.

[5] Chin-I Lee, (May 2011) "Securing Vehicular Ad Hoc Networks: A Research Survey" Ling Tung University.

[6] Bhakthavathsalam, R. Nayak, S., (May 2011) "Operational inferences on VANETs in 802.16e and 802.11p with improved performance by Congestion Alert", Consumer Communications and Networking Conference (CCNC), IEEE.

[7] CanuMobiSim available at http://canu.informatik.uni-stuttgart.de/mobisim/ Visited in May 2011.

[8] GloMoSim available at http://pcl.cs.ucla.edu/projects/glomosim/Visited in Jan. 2011.

[9] VanetMobiSim available at http://vanet.eurecom.fr/ Visited in Jan. 2011.

[10] J. Ott and D. Kutscher, (2004) "Drive-thru Internet: IEEE 802.11b for Automobile Users", In IEEE Infocom.

[11] S. Jaap, M. Bechler, L. Wolf, (June 2005) "Evaluation of Routing Protocols for Vehicular Ad Hoc Networks in City Traffic Scenarios," Proceedings of the 5th International Conference on Intelligent Transportation Systems (ITS) Telecommunications.

[12] B. Ramakrishnan, Dr. R. S. Rajesh, R. S. Shaji, (November 2010) “ An Intelligent Routing Protocol for Vehicle safety communication in Highway Environments”, Journal of Computing , Volume 2, Issue 11.

[13] Carolina Tripp Barba, Karen Ornelas, Mónica Aguilar Igartua, (October 2010) "Performance Evaluation of a Hybrid Sensor and Vehicular Network to Improve Road Safety", 7th ACM PEWASUN 2010, Bodrum, Turkey.

[14] Automotive industry in India available at http://en.wikipedia.org/wiki/Automotive_industry_in_India Visited in May 2011.

[15] SUN Xi, LI Xia-miao, (Oct. 2008) "Study of the Feasibility of VANET and its Routing Protocols", Wireless Communications, Networking and Mobile Computing, 4th International Conference.

[16] J. J. Blum, A. Eskandarian, and L. J. Hoffman, (Dec. 2004) "Challenges of Inter vehicle Ad Hoc Networks", IEEE Transactions on Intelligent Transportation Systems, Vol. 5. No. 4”.

[17] J.Blum, A. Eskandarian, and L. Hoffman, (2003) "Performance Characteristics of Inter- Vehicle Ad Hoc Networks", The IEEE $6^{\text {th }}$ International Conference on Intelligent Transportation Systems, Shanghai, China, page 114-119.

[18] Yun-Wei Lin, Yuh-Shyan Chen and Sing-Ling Lee, (2010) "Routing in Vehicular Ad Hoc Networks: A Survey and Future Perspectives," Journal of Information Science and Engineering.

[19] Sajjad Ali \& Asad Ali, (2009) "thesis - Performance Analysis of AODV, DSR and OLSR in MANET", Department of Electrical Engineering with emphasis on Telecommunication Blekinge Institute of Technology, Sweden.

[20] R.S. Raw, S. Kumar, S. Das, (2010 ) "An Efficient Position-Based Routing Protocol for Vehicular Ad Hoc Network in City Traffic Scenarios”, RISTEP-2010, NIT Srinagar, India. 
International Journal of Wireless \& Mobile Networks (IJWMN) Vol. 3, No. 4, August 2011

[21] Fan Li and Yu Wang, "Routing in Vehicular Ad Hoc Networks: A Survey," IEEE Vehicular Technology Magazine.

[22] Bilal Mustafa, Umar Waqas Raja, (June-2010) "Issues of Routing in VANET", Thesis no: MCS2010-20, Sweden.

[23] WU Zhuang, YANG Yang, GUO Xun and AN Jianwei, (Jan. 2010) "Analysis of Collision Probability in IEEE 802.11 Based VANETs", Chinese Journal of Electronics, Vol.19.

[24] C. Hernández-Goya, P. Caballero-Gil, J. Molina-Gil, C. Caballero-Gil, (2009) "Cooperation requirements for packet forwarding in vehicular ad-hoc networks (VANETs)", ACM International Conference Proceeding Series, Vol. 433.

[25] Sandhaya Kohli, Bandanjot Kaur, Sabina Bindra, (Apr 2010) “A comparative study of Routing Protocols in VANET", RIMT-IET, 10.

[26] NS-2.34, available at http://www.isi.edu/nsnam/ns Visited in May-2011.

[27] Kevin Fall, Kannan Varadhan, (May 9, 2010) “The ns Manual”, The VINT Project, available at http://www.isi.edu/nsnam/ns/ns-documentation.

\section{Authors}

Prof. Aditya Shastri is presently Professor of Computer Science \& Vice Chancellor at Banasthali University. He received his M.Sc. (Tech.) Computer Science \& M.Sc. (Hons.) Maths degree from BITSPilani, India before completing his Ph.D. at Massachusetts Institute of Technology, Cambridge USA in 1990. His research interests are in Discrete Mathematics, Graph Theory and Mobile Computing. He has written more than 50 research papers in journals of repute and authored 5 textbooks. Of late, he is engaged in the development of Banasthali University as its Chief Executive Officer and Chief Academic Officer.

Dr. Reena Dadhich is presently working as a Associate Professor and Head of the Department of Master of Computer Applications at Engineering College Ajmer, India. She received her Ph.D. (Computer Sc.) and M.Sc. (Computer Sc.) degree from Banasthali University, India. Her research interests are Algorithm Analysis \& Design, Wireless Ad-Hoc Networks and Software Testing. She has more than 12 years of teaching experience. She is working as an Editorial Board Member / Reviewer/Committee member of various International Journals and Conferences. She has written many research papers and authored 2 books.

Ramesh C. Poonia is research scholar of computer networking has comprehensive knowledge in the field of Information Technology. Post Master Degree competence makes him much suitable in presenting the matter in the present form. Apart from teaching currently author is pursuing Ph.D. from Banasthali University which will open new doors for creativity in the field of Computer Science. 